\title{
Theory of cw Raman oscillation in optical fibers
}

\author{
John AuYeung and Amnon Yariv \\ California Institute of Technology, Pasadena, California 91125 \\ (Received 25 November 1978)
}

\begin{abstract}
A theory for Raman fiber laser oscillators is presented. The Stokes output and the residual pump power are shown to be calculable from a knowledge of the input pump power and the fiber characteristics. The evolution of the pump and the Stokes waves along the fiber are also obtained. The threshold pump power and the oscillation condition are considered. Pump depletion is incorporated in the analysis and is shown to be the dominant saturation effect present in a Raman fiber laser.
\end{abstract}

\section{INTRODUCTION}

A low loss optical fiber is capable of guiding electromagnetic waves over long distances with little attenuation, confining it simultaneously to a small cross-sectional area. This results in a long interaction region and a large optical field intensity. This is a favorable situation for studying nonlinear optical phenomena. It was shown that threshold powers of a few watts are sufficient to generate stimulated Raman scattering (SRS) and stimulated Brillouin scattering (SBS). ${ }^{1-8}$ New coherent sources in the visible and the infrared frequencies were demonstrated recently by making use of the stimulated scattering effects in fibers. ${ }^{5-10}$ Fiber Raman lasers, oscillating at wavelengths tunable between 1.1 and $1.5 \mu \mathrm{m}$, are especially useful in probing the properties of glass fibers ${ }^{10,11}$ which have minimum absorption and dispersion in this wavelength region. The research in this area has been largely experimental and a comprehensive theory of $\mathrm{cw}$ Raman fiber oscillators has been needed for some time. Several authors have investigated the problem of a single-pass forward or backward scattering process. ${ }^{12,13}$ The effect of pump depletion due to the down conversion to Stokes waves was neglected in their analyses. In a recent article, ${ }^{14}$ we incorporated both the pump depletion and the spontaneous scattering effects in the treatment of amplification by forward SRS in a single-mode fiber. It was shown that the nondepleted pump approximation is invalid for low loss fibers. The consideration of pump depletion is even more critical in understanding the oscillations of the Stokes and the Brillouin shifted waves. A simplified oscillator theory has been presented that does account for pump depletion in the case of $\mathrm{cw}$ Brillouin ring oscillators. ${ }^{15} \mathrm{We}$ present here an analysis of the cw stimulated Raman oscillation in optical fibers. A method is derived to calculate the steady-state output power of the SRS wave when the input pump power and the relevant parameters of the fiber resonant cavity are known. Good agreement with published experimental results is obtained. The evolution of the pump and the Stokes waves along the fiber are calculated. An expression for the threshold pump power is obtained. The oscillation condition and higher-order Stokes waves generation are discussed.

\section{ANALYSIS}

Consider a situation as depicted in Fig. 1. A laser beam is coupled by a lens $L_{1}$ into a single mode fiber inside a FabryPerot resonator. The presence of this pump beam inside the fiber medium gives rise to a gain for an electromagnetic wave at a different (Stokes) frequency because of the stimulated Raman effect. Mirrors $M_{1}$ and $M_{2}$ are highly reflective at this
Stokes frequency. As the pump power is increased, the round-trip gain for the Stokes wave will eventually exceed the loss and result in oscillation. The prism couples out a small fraction of the Stokes wave and also prevents the residual pump beam from being reflected back into the pumping laser. A large pump to Stokes wave conversion is usually attained, and hence the loss of the unused pump power in this geometry is unimportant.

There are three waves propagating inside the fiber. A pump wave of frequency $\nu_{p}$ travels in the forward direction. It generates a forward and a backward traveling Stokes wave at a frequency $\nu_{s}$, which is down shifted from $\nu_{p}$ by the vibrational frequency of the molecular medium. A classical treatment ${ }^{16}$ of the stimulated Raman scattering process shows that the optical powers of the three waves obey the following equations:

$$
\begin{gathered}
\frac{d P_{p}}{d z}=-\alpha P_{p}-\frac{\nu_{p}}{\nu_{s}} \frac{g}{A} P_{p}\left(P_{f}+P_{b}\right), \\
\frac{d P_{f}}{d z}=-\alpha P_{f}+\frac{g}{A} P_{p} P_{f},
\end{gathered}
$$

and

$$
\frac{d P_{b}}{d z}=\alpha P_{b}-\frac{g}{A} P_{p} P_{b} .
$$

$P_{p}, P_{f}$, and $P_{b}$ refer to the pump wave power, the forward Stokes wave power, and the backward Stokes wave power, respectively. The first term on the right side of each equation is due to the linear absorption in the fiber medium, where we have assumed the absorption coefficient $\alpha$ to be identical for the three waves. The second terms on the right sides of the equations describe the nonlinear stimulated process and couple the three waves together. $A$ is the effective core area of the single mode fiber and $g$ is the Raman gain constant in units of $\mathrm{cm} / \mathrm{W} . z$ is the distance measured along the fiber with $z=0$ being the end at which the pump wave is injected into the fiber. We assume a cw steady-state situation, with the Stokes wave oscillation already established. The problem of how the oscillation builds up from the spontaneous Raman scattering is not considered here. During oscillation, the intracavity Stokes-photon flux is so high that stimulated scattering is the dominant photon-conversion process. We are therefore justified in neglecting the spontaneous emission terms in our equations. We note that because of the depolarization effects inside a fiber, a slightly smaller Raman gain constant will have to be used in the above equations. 


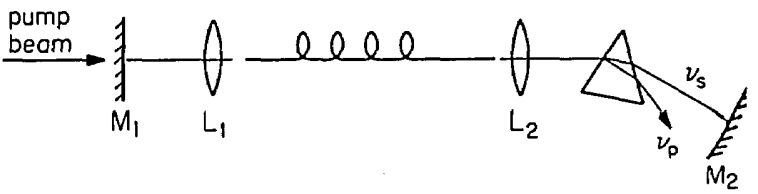

FIG. 1. Typical experimental setup.

The boundary conditions of the waves are:

$$
\begin{gathered}
P_{p}(z=0) \equiv P_{p o}, \\
P_{f}(z=0) \equiv P_{f o}=R_{1} P_{b}(z=0) \equiv R_{1} P_{b o},
\end{gathered}
$$

and

$$
P_{b}(z=L) \equiv P_{b L}=R_{2} P_{f}(z=L) \equiv R_{2} P_{f L} .
$$

$L$ is the total length of the fiber and $P_{p o}$ is the amount of pump power actually coupled into the input end of the fiber. $R_{1}$ and $R_{2}$ are the effective power reflectivities relating the counterpropagating Stokes waves at the two fiber ends; the values of the reflectivities have taken into account the lens coupling loss, the fiber end loss, the prism loss, and the mirror transmission loss on each side of the fiber.

As the Stokes wave propagates along the fiber, it is attenuated exponentially as $\exp (-\alpha z)$ due to scattering and linear absorption in the medium. At the same time, it is amplified exponentially as $\exp \left[(g / A) \int_{o}^{z} P_{p}\left(z^{\prime}\right) d z^{\prime}\right]$ due to the stimulated Raman effect. The evolution of each Stokes wave is governed by the same physical mechanisms; and Eqs. (2) and (3) differ only because of the counterpropagating nature of the two waves. It follows directly from (2) and (3) that

$$
P_{f}(z) P_{b}(z)=P_{f o} P_{b o}=P_{f L} P_{b L}=K,
$$

where $K$ is a $z$-independent constant- Using the boundary conditions in (5) and (6), it follows directly from (7) that

$$
P_{f o} / P_{f L}=P_{b L} / P_{b o}=\left(R_{1} R_{2}\right)^{1 / 2} .
$$

This equation specifies a relation between the end values of each Stokes wave. An alternative relation can be obtained by integrating (2) and (3), with the result

$$
\frac{P_{f o}}{P_{f L}}=\frac{P_{b L}}{P_{b o}}=\exp \left[\alpha L-\frac{g}{A} \int_{0}^{L} P_{p}(z) d z\right] .
$$

Equating the above two relations immediately leads to an oscillation condition for the Stokes waves, namely

$$
\left(R_{1} R_{2}\right)^{1 / 2} \exp \left[-\alpha L+\frac{g}{A} \int_{0}^{L} P_{p}(z) d z\right]=1 .
$$

Equation (10) is a statement of the fact that for oscillation the round-trip gain of the Stokes wave is equal to its loss which consists of absorption inside the fiber medium and other cavity losses accounted for by $R_{1}$ and $R_{2}$. The pump "profile" $P_{p}(z)$ will adjust itself so that (10) is satisfied, even when the pump power exceeds the threshold value for oscillation. This is the basis of gain saturation in a Raman oscillator.

The Stokes output power is proportional to $P_{f L}$ and no distinction shall be made between them hereafter. We now proceed to solve for the Stokes output power $P_{f L}$ and the residual pump power $P_{p}(z=L) \equiv P_{p L}$ for a given input pump power $P_{p o}$. Substitute $P_{f}=K / P_{b}$ into (1), divide (3) by (1), and then integrate the resulting equation. We obtain

$$
\begin{array}{r}
P_{p}(z) P_{b}(z) \exp \left\{-\frac{g \nu_{p}}{\alpha A \nu_{s}}\left[\frac{\nu_{s}}{\nu_{p}} P_{p}(z)+P_{f}(z)-P_{b}(z)\right]\right\} \\
=P_{p o} P_{b o} \exp \left\{\frac{-g \nu_{p}}{\alpha A \nu_{s}}\left[\frac{\nu_{s}}{\nu_{p}} P_{p o}+P_{f o}-P_{b o}\right]\right\}=\mathrm{C} .
\end{array}
$$

$\mathrm{C}$ is a constant and (11) describes a parametric trajectory in the space of $P_{p}, P_{f}$, and $P_{b}$. It relates the three waves at an arbitrary $z$ within the fiber. In particular, at $z=L$, (11) can be reduced to

$$
P_{f L}=\frac{\ln \left[\left(R_{1} R_{2}\right)^{1 / 2} P_{p L} / P_{p o}\right]+(g / \alpha A)\left(P_{p o}-P_{p L}\right)}{(g / \alpha A)\left(\nu_{p} / \nu_{s}\right)\left\{1-R_{2}-\left(R_{1} R_{2}\right)^{1 / 2}\left[1-\left(1 / R_{1}\right)\right]\right\}} .
$$

Equations (5), (6), and (8) are used in obtaining (12). We have thus expressed the Stokes output power $P_{f L}$ as a function of the input and residual pump powers, $P_{p o}$ and $P_{p L}$, respectively. Equation (12) provides one of the two equations needed for solving $P_{f L}$ and $P_{p L}$.

It can be shown from (1), (2), and (3) that

$$
\frac{d v}{d z}=-\alpha u,
$$

where $v=\left(\nu_{s} / \nu_{p}\right) P_{p}+P_{f}-P_{b}$ and $u=\left(\nu_{s} / \nu_{p}\right) P_{p}+P_{f}+P_{b}$. This is a statement of the fact that the total photon number is conserved during the Raman process and the only loss mechanism is due to the absorption by the fiber medium. We note that solving for $u, v$, and $K$ [defined in (7)] is equivalent to solving $P_{p}, P_{f}$, and $P_{b}$, each of which can be expressed in terms of the former three parameters. In particular, when (11) is written in terms of $u, v$, and $K$, and using (13) to eliminate $u$ in the resulting equation, leads to the differential equation

$$
\begin{aligned}
\frac{d v}{d z}=-\left\{\alpha^{2} v^{2}+4 \alpha^{2} K+4 \alpha^{2}\right. & \frac{\nu_{s}}{\nu_{p}} P_{p o} P_{b o} \\
& \left.\times \exp \left[\frac{-g \nu_{p}}{\alpha A \nu_{s}}\left(v_{o}-v\right)\right]\right\}^{1 / 2},
\end{aligned}
$$

where $v_{o}$ is $v(z=0)$. Integrating (14) results in

$$
\begin{aligned}
\int_{v(z)}^{v_{0}} d v^{\prime}\left\{\alpha^{2} v^{\prime 2}+4 \alpha^{2} K+4 \alpha^{2} \frac{\nu_{s}}{\nu_{p}} P_{p o} P_{b o}\right. \\
\left.\times \exp \left[\frac{-g \nu_{p}}{\alpha A \nu_{s}}\left(v_{o}-v^{\prime}\right)\right]\right\}^{-1 / 2}=z .
\end{aligned}
$$

This equation will enable us later on to calculate $P_{p}, P_{f}$, and $P_{b}$ within the fiber. At $z=L$, we have

$$
\begin{aligned}
\int_{v_{L}}^{v_{o}} d v^{\prime}\left\{\alpha^{2} v^{\prime 2}+4 \alpha^{2} K+4 \alpha^{2} \frac{\nu_{s}}{\nu_{p}} P_{p o} P_{b o}\right. \\
\left.\times \exp \left[\frac{-g \nu_{p}}{\alpha A \nu_{s}}\left(v_{o}-v^{\prime}\right)\right]\right\}^{-1 / 2}=L,
\end{aligned}
$$

where $v_{L} \equiv v(z=L) . \quad v_{0}, v_{L}$, and $K$ can all be expressed in terms of $P_{f L}$ and $P_{p L}$, and (16) is the second equation, which together with (12) will allow us to solve for the two unknowns, $P_{f L}$ and $P_{p L}$. The integral in (16) can be performed by straightforward numerical techniques. It is also discovered that a linear approximation of the exponential term in the integrand makes (16) integrable and the $P_{f L}$ and $P_{p L}$ values thus calculated agree closely with the exact values obtained by the numerical technique. A method has thus been derived 
that allows the prediction of the Stokes output and the residual pump powers, when the input pump power and the fiber-resonator parameters are known. We will illustrate the usefulness of this method by applying it to a typical example. It will also be shown that in addition to finding $P_{f L}$ and $P_{p L}$, our treatment enables us to calculate $P_{p}(z), P_{f}(z)$, and $P_{b}(z)$ at all $z$ 's along the fiber.

\section{EXAMPLE}

To illustrate how to make use of the results derived in the preceding section, we shall consider the case of a recently reported high efficiency cw Raman-fiber oscillator. ${ }^{5}$ The experimental setup was the same as that depicted in Fig. 1. From the provided data, $g=0.9 \times 10^{-11} \mathrm{~cm} / \mathrm{W}, \alpha=3.9 \times 10^{-5}$ $\mathrm{cm}^{-1}, \mathrm{~A}=9.9 \times 10^{-8} \mathrm{~cm}^{2}, c / \nu_{p}=0.5145 \mu \mathrm{m}, c / \nu_{s}=0.5280 \mu \mathrm{m}$, $R_{1}=0.7$, and $R_{2}=0.4$. The pumping power $P_{p o}$ was $4 \mathrm{~W}$. A $P_{f L}$ is calculated according to (12) for an assumed value of $P_{p L}$. This pair of $P_{p L}$ and $P_{f L}$ in conjunction with Eqs. (5), (6) and $(8)$ is then used to find the corresponding $v_{o}$ and $v_{L}$ which, in turn, when substituted into (16), determine $L$. This $L$ will be the length of a fiber having these particular $P_{p L}$ and $P_{f L}$ values at its output surface for the given pump power and resonator parameters. Hence, by varying $P_{p L}$ from zero to $P_{p o}$, we are able for each value of $P_{p L}$ to calculate the corresponding Stokes output power and also determine the fiber length required to observe such an output. According to (12), some $P_{p L}$ values, which are close to either $P_{p o}$ or zero, result in negative values for $P_{f L}$. This occurs either when $L$ is too short to provide a sufficient interaction length, or when $L$ is too long so that attenuation per pass is too large. In both cases, there is no oscillation and they will not concern us here. We have plotted in Fig. 2, using the above mentioned data, $P_{p L}$ and $P_{f L}$, for different pump power values with $L$ along the horizontal axis. In the figure the $P_{p L}$ and $P_{f L}$ at a particular $L$ value are the predicted residual pump and Stokes output powers if an experiment is done using a fiber length of that $L$ value. In the reported experiment, the length of the fiber used was $100 \mathrm{~m}$. At a pump power of $4 \mathrm{~W}$ the estimated Stokes power after lens $L_{2}$ (see Fig. 1) was reported to be $2 \mathrm{~W}$, corresponding to a $P_{f L}$ of $2.3 \mathrm{~W}$. $P_{p L}$ was estimated to be $0.5 \mathrm{~W}$. Our calculation

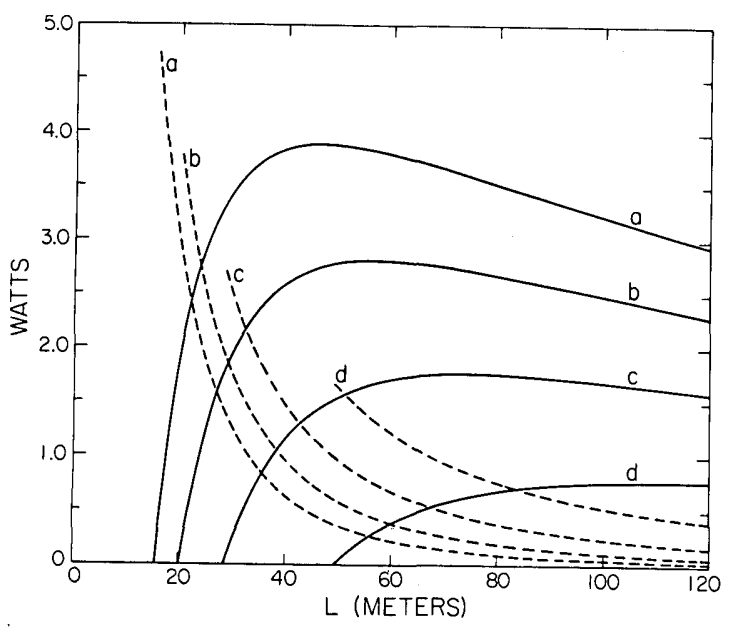

FIG. 2. Solid lines are the Stokes output powers and the broken lines are the residual pump powers. The input pump powers are (a) $5 \mathrm{~W}$, (b) $4 \mathrm{~W}$, (c) $3 \mathrm{~W}$, and (d) $2 \mathrm{~W}$.

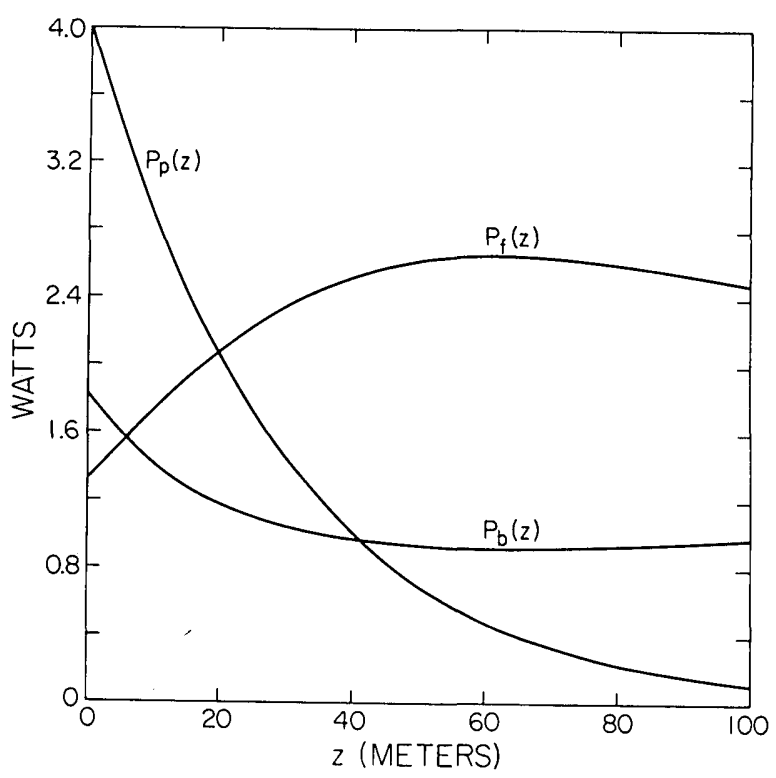

FIG. 3. The pump and the Stokes powers inside the $100-\mathrm{m}$ long fiber at a $4 \mathrm{~W}$ pump power input.

shown in Fig. 2 indicates that for $\mathrm{L}=100 \mathrm{~m}$ and $P_{p o}=4 \mathrm{~W}, P_{f L}$ is $2.5 \mathrm{~W}$ and $P_{p L}$ is $0.1 \mathrm{~W}$. The agreement between the calculated and the reported values is quite reasonable.

In addition to predicting the Stokes output and the residual pump powers, it is also possible to calculate for a given fiber length $L, P_{p}, P_{f}$, and $P_{b}$ at each point along the fiber. For example, in the above-mentioned case for which $P_{p L}$ and $P_{f L}$, and hence $K, v_{o}$, and $v_{L}$ have been calculated, a $v(z)$ value is substituted into (15) to find $z$. This $z$ is then the distance measured along the fiber where $v(z)$ has that particular value. Moreover, $u(z)$ at this point is also known as provided by (13) and (14). Consequently, $P_{p}, P_{f}$, and $P_{b}$ are obtained in terms of the $v(z), u(z)$, and $K$ values. Therefore, by varying $v(z)$ from $v_{o}$ to $v_{L}$, we are able to determine $P_{p}(z), P_{f}(z)$, and $P_{b}(z)$ for each $z$ calculated. Figure 3 shows the evolution of the three powers along the $100 \mathrm{~m}$ fiber of our example for a $4 \mathrm{~W}$ input pump power. The rapid depletion of the pump wave due to the conversion to Stokes photons is evident.

\section{DISCUSSION}

It is apparent, as shown in Fig. 2, that for a given input pump power there is an upper limit to the amount of Stokes output power obtainable from a fiber Raman oscillator. According to (12), when $P_{p L}=\alpha A / g$, the Stokes output is maximum and is equal to

$$
\begin{aligned}
& P_{f L}(\max )= \\
& \quad \frac{\ln \left[\left(R_{1} R_{2}\right)^{1 / 2}\left(\alpha A / g P_{p o}\right)\right]+(g / \alpha A) P_{p o}-1}{(g / \alpha A)\left(\nu_{p} / \nu_{s}\right)\left\{1-R_{2}-\left(R_{1} R_{2}\right)^{1 / 2}\left[1-\left(1 / R_{1}\right)\right]\right\}} .
\end{aligned}
$$

The correct fiber length, $L_{o p t}$, to obtain this maximum Stokes output, can be determined from (16), since $P_{p L}$ and $P_{f L}$ are known. A fiber length that is shorter than $L_{\text {opt }}$ yields smaller Stokes output power because the interaction length is shorter, resulting in smaller gain per pass. A fiber length longer than $L_{\text {opt }}$ also results in smaller Stokes output power because the loss per pass is larger. $L_{o p t}$, at $4-\mathrm{W}$ pumping power of our example, is about $57 \mathrm{~m}$. Using a fiber of this length should 
produce, at the specified pump power, higher Stokes output than what was reported. When designing a fiber Raman resonator which is intended to be pumped at a constant input power, $L_{\text {opt }}$ is obviously an important paramoter though, as shown in Fig. 2, small deviations from this optimum value are easily tolerated.

We derived in (10) the oscillation condition for the fiber Raman laser. When the pump power is increased from zero to a value such that this condition is barely satisfied, the oscillating intracavity Stokes power is very small. At this threshold situation, the pump power will behave as $P_{p t} \exp (-\alpha z)$ where $P_{p t}$ is the threshold input pump power. The exponential behavior of the pump follows directly from (1), the pump beam being attenuated only by the absorption of the medium in the absence of any Stokes wave. Upon substituting this threshold pump power behavior into (10), the required threshold pump input for oscillation is obtained as

$$
P_{p t}=\frac{\alpha A}{g}\left[\frac{\alpha \mathrm{L}-(1 / 2) \ln \left(R_{1} R_{2}\right)}{1-\exp (-\alpha L)}\right] .
$$

This is calculated to be $1.35 \mathrm{~W}$ when the data provided in the reported experiment of our example is used. The actual threshold value measured in the experiment was reported to be $1 \mathrm{~W}$.

An interesting feature which results from (10) is that above threshold $\left(P_{p o}>P_{p t}\right) \int_{0}^{L} P_{p}(z) d z$ is independent of the input pump power $P_{p o}$. The total absorption of the pump power by the fiber is then

$$
\alpha \int_{0}^{L} P_{p}(z) d z=P_{p t}[1-\exp (-\dot{\alpha} L)] .
$$

When the input pump power exceeds the threshold value, saturation effect keeps the single pass gain constant so as to maintain the oscillation condition described by (10). This is achieved because, as the Stokes intensity increases, the nonlinear coupling also increases, resulting in an extremely fast draining of the photons from the pump wave. The pump wave becomes very much depleted after a short distance so that the Stokes wave gain in the rest of the fiber is reduced and the overall gain per pass remains clamped. Thus pump depletion is the dominant saturation effect in the fiber Raman laser.

In our analysis, we have neglected the higher-order Stokes waves generation, which arises because the forward and backward propagating Stokes waves inside the fiber can act as pump waves to generate their own down-shifted Stokes waves. For simplicity, we shall consider only the second-order forward Stokes waves and demonstrate that it is insignificantly small. The second-order backward Stokes wave is of the same order of magnitude as its forward counterpart, while the third- and higher-order Stokes waves are even smaller. The power of the second-order forward Stokes wave, denoted by $P_{f}^{\prime}$, obeys the equation

$$
\frac{d P_{f}^{\prime}}{d z}=-\alpha P_{f}^{\prime}+\frac{g^{\prime}}{A} P_{f}^{\prime}\left(P_{f}+P_{b}\right)
$$

the solution of which at $z=L$ is

$$
P_{f L}^{\prime}=P_{f o}^{\prime} \exp \left\{-\alpha L+\frac{g^{\prime}}{A} \int_{0}^{L}\left[P_{f}(z)+P_{b}(z)\right] d z\right\}
$$

Equations (2) and (3) that describe $P_{f}$ and $P_{b}$ will have additional loss terms to account for the second-order nonlinear effect. However, the original solutions of (2) and (3) will be the upper limits to the solutions when the second order effect is incorporated. Therefore, for our purpose, which is to estimate the maximum second-order Stokes wave that can possibly be generated, it is adequate to simply use the previous calculated values of $P_{f}$ and $P_{b}$ in (21). Remembering our definition of $u$,

$$
\begin{aligned}
\int_{0}^{L}\left[P_{f}(z)+P_{b}(z)\right] & d z \\
= & \int_{0}^{L} u(z) d z-\frac{\nu_{s}}{\nu_{p}} \int_{0}^{L} P_{p}(z) d z .
\end{aligned}
$$

Upon using (13), (18), and (19), Eq. (22) becomes

$$
\begin{aligned}
\int_{0}^{L}\left[P_{f}(z)+P_{b}(z)\right] d z= & \frac{1}{\alpha}\left[v_{o}-v_{L}\right] \\
& -\frac{\nu_{s}}{\nu_{p}} \frac{A}{g}\left[\alpha L-\frac{1}{2} \ln \left(R_{1} R_{2}\right)\right],
\end{aligned}
$$

which is expressed in terms of previously calculated values. Thus $P_{f L}^{\prime}$ can be obtained from (21) and (23). Since there is actually no finite $P_{f o}^{\prime}$, and any second-order Stokes power build-up is due to amplified spontaneous scattering, we shall arbitrarily assume one photon per mode as a fictitious input for $P_{f}^{\prime}$. Using a Lorentzian gain constant with a $400 \mathrm{~cm}^{-1}$ linewidth and the data provided in our example, $P_{f L}^{\prime}$ is estimated to be only about $30 \mu \mathrm{W}$. This justifies our neglect of the higher-order Stokes waves in the analysis.

\section{CONCLUSION}

We have presented a detailed treatment of a stimulated Raman fiber laser. We have demonstrated how the Stokes output power and the residual pump power can be calculated from a known input power and the resonator parameters. In addition, the evolution of the pump and the Stokes waves along the fiber are calculated. Our result is compared with published experimental data and reasonable agreement is obtained. The threshold pump power and the oscillation condition at and above threshold are discussed. Higher-order Raman effect is shown to be negligible, confirming that pump depletion is the dominant saturation effect. The extension of the above analysis to the case of SBS oscillation will be discussed in a future publication.

\section{ACKNOWLEDGMENT}

This research was supported by the Air Force Office of Scientific Research.

${ }^{1}$ E. P. Ippen, Appl. Phys. Lett. 16, 303 (1970).

2 E. P. Ippen and R. H. Stolen, Appl. Phys. Lett. 21, 539 (1972).

3 J. Stone, Appl. Phys. Lett. 26, 163 (1975).

${ }^{4}$ K. O. Hill, B. S. Kawasaki, and D. C. Johnson, Appl. Phys. Lett. 29, 181 (1976).

${ }^{5}$ C. Lin, R. K. Jain, and R. H. Stolen, J. Opt. Soc. Am. 67, 250A (1977); R. K. Jain, C. Lin, R. H. Stolen, W. Pleibel, and P. Kaiser, Appl. Phys. Lett. 30, 162, (1977).

${ }^{6}$ K. O. Hill, B. S. Kawasaki, and D. C. Johnson, J. Opt. Soc. Am. 66, 1114A (1976); D. C. Johnson, K. O. Hill, B. S. Kawasaki and D. 
Kato, Electron. Lett. 13, 53 (1977).

${ }^{7}$ R. K. Jain, C. Lin, R. H. Stolen and A. Ashkin, Appl. Phys. Lett. 31, 89 (1977).

${ }^{8}$ C. Lin, R. H. Stolen, W. G. French, and T. G. Malone, Opt. Lett. 1,96 (1977).

${ }^{9}$ R. H. Stolen, C. Lin, and R. K. Jain, Appl. Phys. Lett. 30, 340 (1977).

${ }^{10}$ C. Lin, R. H. Stolen, and L. G. Cohen, Appl. Phys. Lett. 31, 97 (1977).
11 L. G. Cohen and C. Lin, Appl. Opt. 16, 3136 (1977).

12 R. G. Smith, Appl. Opt. 11, 2489 (1972).

13 F. Capasso and P. D. Porto, J. Appl. Phys. 47, 1472 (1976).

14 J. AuYeung and A. Yariv, IEEE J. Quantum Electron. QE-14, 347 (1978).

15 D. C. Johnson, K. O. Hill and B. S. Kawasaki, Radio Sci. 12, 519 (1977).

16 A. Yariv, Quantum Electronics, 2nd ed. (New York, Wiley, 1975).

\title{
Analysis of optical channel waveguides and directional couplers with graded-index profile
}

\author{
Toshiaki Suhara, Yuichi Handa, Hiroshi Nishihara, and Jiro Koyama \\ Osaka University, Department of Electronics, Faculty of Engineering, Yamada-kami, Suita, Osaka 565, Japan
}

(Received 28 December 1978)

\begin{abstract}
A theoretical analysis of graded-index optical channel waveguides and directional couplers fabricated by the beam-writing technique is presented. The analysis is based on a combination of the effective-index-of-refraction method and the WKB method, which is shown to apply for directional couplers as well as for channel waveguides, when special care is taken in connecting the field at the gap region. The mode dispersion characteristics and the field profile of a channel waveguide were derived. The conversion length of a directional coupler was also determined as a function of the coupler parameters and the mode order. The guided mode field has long evanescent tails outside the guide, and the positions of the turning points are located further away from the guide center for higher-order modes. Accordingly, the conversion length of a coupler increases slowly with the waveguide spacing and exhibits a strong dependence on the transverse mode order. The results are summarized in a few practically useful design charts by employing the normalizations of the device parameters. Some design examples are given and the fabrication tolerances are discussed.
\end{abstract}

\section{INTRODUCTION}

Optical channel waveguides and directional couplers are among the most important elements in constructing integrated optical circuits and have been actively studied. In particular, devices with a step refractive-index profile fabricated by lithography and etching technique have been extensively analyzed. ${ }^{1,2}$ The fundamental properties are well understood and the design techniques are established. A number of experimental works have been performed as well. ${ }^{3-5}$

On the other hand, the recent developments in waveguide materials and fabrication techniques have made it possible to produce channel waveguides and directional couplers with a graded refractive-index profile. There have been great interests in such devices because of their excellent waveguiding characteristics, easy fabrication and advantages in giving various additional functions. However, optical devices with a graded index profile are quite difficult to analyze. For this reason, they used to be designed by employing an approximation to a step index profile. An accurate analysis for the graded index profile remains to be developed.

A channel waveguide fabricated by the selective diffusion of metal in an electro-optical crystal has an index profile graded mainly in the direction of the substrate thickness. The wave-guiding mode characteristics were analyzed by means of the effective-index-of-refraction method by Hocker et al. ${ }^{6}$ Taylor employed a numerical method to analyze the mode dispersion characteristics taking into account the waveguide broadening by the transverse diffusion. ${ }^{7}$ As for a directional coupler, Taniuchi et al. applied the multiple layer decomposition method to a two-dimensional model and compared the coupling characteristics of couplers with graded and step index profiles. 8 However, detailed analysis giving a guide for designing couplers with a graded three-dimensional index profile seems not yet to have been reported.

The authors have developed a direct electron-beam writing technique for the fabrication of micro-optical components, making use of the electron-beam-induced refractive-index increase in chalcogenide amorphous semiconductor thin films. ${ }^{9-12}$ By this technique, one can fabricate channel waveguides and directional couplers with an index profile graded in the transverse direction along the plane of the waveguide. Channel guides and couplers with similar index profile were made by the laser beam writing technique in photosensitive waveguide materials. ${ }^{13,14}$ The design theory for this type of device, however, is not well established, and a considerable mismatch has been reported between the experimentally obtained coupling characteristics and those predicted theoretically by using the step index approximation. ${ }^{14}$

The purpose of this paper is to analyze optical channel waveguides and directional couplers with graded index profile fabricated by the electron- or laser-beam writing technique and establish the design procedure. In the following sections, we first show that the devices can be analyzed by a combina- 